\title{
Star image identification uninfluenced by rotation
}

\author{
Jiang $\mathrm{Di}^{1, \mathrm{a}}$, Zhang $\mathrm{Ke}^{1}$, Lv Meibo ${ }^{1}$ \\ ${ }^{1}$ Nortwestern Polytechnical University, Xi'an, China \\ a cindyjiangdi@sina.com
}

Key words: image identification; rotation; star sensor; guide catalog

Abstract: All-sky autonomous star map identification has come up with higher requirement on calculating speed and storage space. Error caused by rotation and location noise influences the performance of star image identification algorithms. So various algorithms include procedure of rotating star image to the position where the algorithm plans. That will have a negative effect on enhancing the performance of identification. This paper presented a recognition method uninfluenced by rotation. The rotation error is eliminated automatically in recognition course. Both position and magnitude information are considered in it. The simulation result indicates that it cost less storage space and the accuracy was increased.

\section{Introduction}

It often occurs that guide stars are rotated off their actual positions when star images are photographed in CCD cameras ${ }^{[1]}$. Thus, most star identification methods come up with procedures including rotating the stars to positions they plan. For example, the grid algorithm. However, the speed of identification will be reduced if we conduct rotation procedures. On the other hand, stars' magnitude is often ignored in the match between images acquired and those in guide star database $^{[2][3]}$. This article presents an algorithm eliminating the rotation in star images observed. It also takes star magnitude into consideration to identify stars in images.

\section{Method descriptions}

The algorithm chooses stars in Hipparcos catalog with magnitude lower than 7 to build a guide catalog for guidance stars. The catalog contains 7548 stars. In the meantime, we assume that the star sensor's field of view is $12^{\circ}$ by $12^{\circ}$, and the size of image plane is 512 by 512 pixels.

\section{Method to generate star images for match}

$$
\left\{\begin{array}{l}
x=\frac{N_{x} \times \cos \delta \sin \left(\alpha_{i}-\alpha\right)}{2 \times \tan \left(\mathrm{FOV}_{x} / 2\right) \times\left(\sin \delta_{i} \sin \delta+\cos \delta_{i} \cos \delta \cos \left(\alpha_{i}-\alpha\right)\right)} \\
y=\frac{N_{y} \times\left(\sin \delta_{i} \cos \delta-\cos \delta_{i} \sin \delta \cos \left(\alpha_{i}-\alpha\right)\right)}{2 \times \tan \left(\mathrm{FOV}_{y} / 2\right) \times\left(\sin \delta_{i} \sin \delta+\cos \delta_{i} \cos \delta \cos \left(\alpha_{i}-\alpha\right)\right)}
\end{array}\right.
$$

Equation (1) is used to calculate the coordinate $(\mathrm{x}, \mathrm{y})$ of each star captured by CCD camera. In Equation (1),

$\delta_{i}$ - right ascension of each star in the star table chosen,

$\alpha_{i}-$ declination of each star in the star table chosen,

$\delta$ - right ascension of the star which is pointed to by axis of view,

$\alpha-$ declination of the star which is pointed to by axis of view. 
The size of image plane is $\mathrm{N}_{\mathrm{x}} \times \mathrm{N}_{\mathrm{y}}$, which is $512^{\times} \times 512$ pixels here,

The star sensor's field of view is $\mathrm{FOV}_{\mathrm{x}} \times \mathrm{FOV}_{\mathrm{y}}$, which is $12^{\circ} \times 12^{\circ}$ here.

The stars' magnitude $M$ is converted to grayscale $G$ according to Equation (2).

$$
G=\frac{255}{2.51^{M-1}}
$$

Figure 1 illustrates an example of star image in guide star database. It is generated via simulation according to Equation (1) and (2) without rotation. In Figure 1, camera's axis of view points to the star of right ascension 41h 20' 43" and declination $1 \mathrm{~h} 2$ ' 54 "' with magnitudes shown as gray scale of star spots.It is simplified so as to be observed more clearly.

\section{Guide templates}

In this article we regard right ascension and declination of each star in Hipparcos catalog in celestial coordinate system as the direction of visual axis and simulate its corresponding star image, then we acquire 7548 star images. They are regarded as guide templates. In fact, the algorithm doesn't simulate images, but only calculate out 7548 matrixes of gray scales corresponding to these star images. So the calculation speed can be enhanced. Then we calculate the features of these templates. We define the feature as below.

$$
P_{k}=\sum_{i=1}^{m_{k}} G(i) \cdot \sqrt{\left(x_{i}-x_{\text {centre }}\right)^{2}+\left(y_{i}-y_{\text {centre }}\right)^{2}}
$$

In Equation (3), $\mathrm{P}_{\mathrm{k}}$ refers to the $\mathrm{k}$ th feature and the sequence numbers of the features are the same with the stars' sequence numbers in guide star catalog;

$i$ is the sequence number of the stars captured in the $k$ th star image, and the sequence number is made from left to right and from top to bottom in the image;

$G(i)$ is the grayscale of the $i$ th star in the image;

$x_{i}$ and $y_{i}$ are star spot's coordinate values in image space coordinate system ;

$x_{c e n t r e}$ and $y_{\text {centre }}$ refer to the centre of the image and they are all 256 here.

That is to say, there are 7548 features as the basis for pattern match afterwards. Moreover, we store the grayscale of central pixel of the images which are taken as guide templates ${ }^{[4]}$. And we give these grayscales the same sequence numbers with the features ${ }^{[5]}$.

We define the star which satisfies the conditions below as the main star in the star image observed. The conditions are: it has the magnitude lower than the other stars within a range. The range is that the angular separation between the main star and the others is less than $p \cdot \mathrm{FOV}_{x}$. In this article, we set $p=0.3$.

It's possible that an observed image will contain several main stars. The algorithm chooses the main star which is nearest to the center of the image as the only main star. Then we translate this star to the center. And with this procedure, the whole image is translated. In order to eliminate the influence of translation to the greatest extent, we expand the image to the size of $1.5 N_{x}$. Thus a new image on the basis of the former one is generated.

After this step, we calculate the feature $P$ of the new image according to Equation (4).

$$
P=\sum_{j=1}^{m} G(j) \cdot \sqrt{\left(x_{j}-x_{\text {centre }}\right)^{2}+\left(y_{j}-y_{\text {centre }}\right)^{2}}
$$

$j$ is the sequence number of the spot which satisfies Equation (5) and has a brightness larger than 0 in the observed star image and the sequence number is made from left to right and from top to bottom in the image; 


$$
\left(x_{j}-x_{\text {centre }}\right)^{2}+\left(y_{j}-y_{\text {centre }}\right)^{2} \leq R^{2}, R=512
$$

$G(j)$ is the grayscale of the $j$ th star in the image;

$x_{j}$ and $y_{j}$ are star spot's coordinate values in image space coordinate system;

$x_{\text {centre }}$ and $y_{\text {centre }}$ refer to the centre of the image and they are all 384 here.

\section{observation templates}

The algorithm presented in this paper constructed an observation template as is illustrated. When calculating the feature of the observation template, the star spot out of the range of Equation (5) won't be included because we intend to find the stars which can match those in one guide template around the only main star. The purpose of expanding the field of view when constructing observation template is to avoid loss in the course of translation.

$P$ is compared with $P_{i}(i=1,2, \mathrm{~L}, k)$ one by one. The guide templates which the algorithm is searching for has feature that is at the same order of magnitude with $P$. We need to set a threshold value for match course. If the difference between $P_{i}$ and $P$ is less than the threshold value, then the guide template corresponding to $P_{i}$ is possible to be the match conclusion. This is the initial filtrate course.

If a guide template has a feature satisfying the threshold value, we compare the grayscale value of the central pixel in its grayscale matrix with the grayscale value of the central pixel in observed star image. The former grayscale value is stored in advance. Theoretically, if the two grayscale values are equal to each other, the algorithm will certify the star corresponding to guide template is the main star in observed star image.

\section{Simulation examples}

In order to verify the algorithm in this article, we generated a star image as is shown in Figure 2 with a random direction of visual axis and assume that it's the image observed through star sensor in operation.

Figure 2 contains more than 10 main stars. The algorithm certified that the only main star is the pixel spot of the coordinate $(226,213)$ in the image. Its grayscale is 5.6480 . Then the image was translated so that the main star could be at the center of the new image generated by translation, as is shown in Figure 3. It corresponds to a grayscale matrix $J$. According to Equation (4), the feature of $J$ is $P=1.7069 \times 10^{4}$.

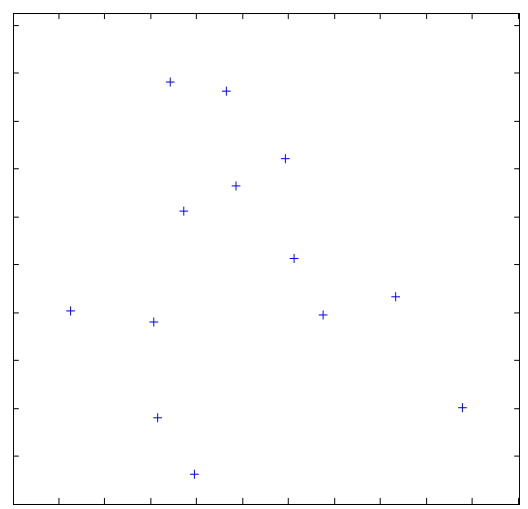

Figure 1 Images in database of guiding stars

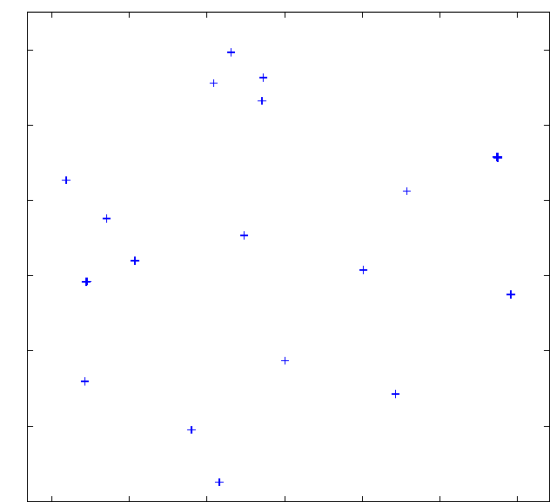

Figure 2 Star image observed by star sensor

In this example, we set 150 as the threshold value. The result of match is the guide template No.1648 with $P_{1648}=1.7020 \times 10^{4}$ and the grayscale value of the central pixel in the 1648 th guide 
template is equal to 5.6480 .

We simulated the star image of No.1648 as is shown in Figure 4 to prove the result is accurate.

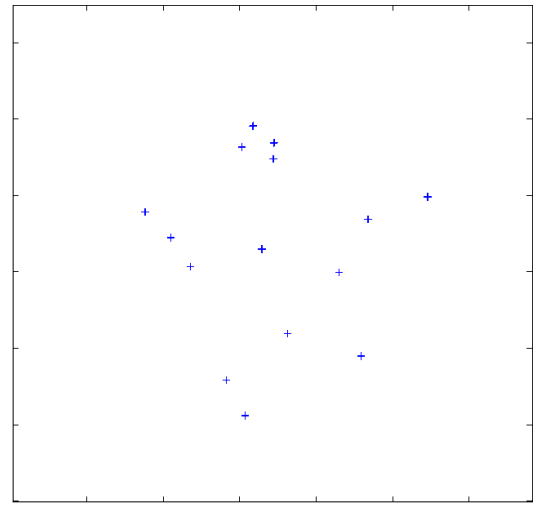

Figure 3 Star image observed after translation

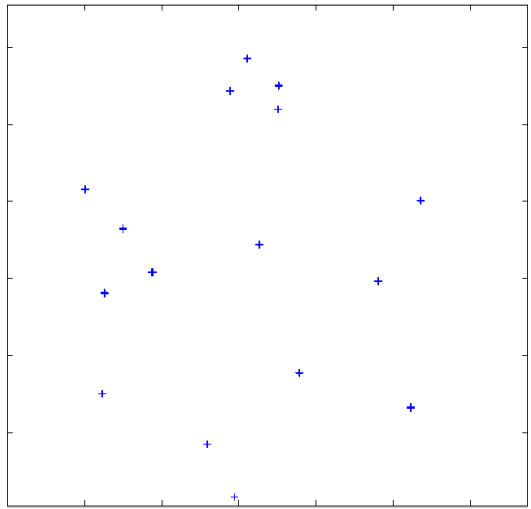

Figure 4 the guide template No.1648

The spot of coordinate $(226,213)$ in Figure 2 is exactly the central star in Figure 4. It means that the star which is represented by the spot of coordinate $(226,213)$ in Figure 2 is the 1648th star in guide catalog. The star has right ascension 11h 24'36" and declination -10h 51'34'.

It can be seen that even if rotation error is added to the image observed, its feature won't change. That is decided by the definition of the feature. So in the course of star image identification, the rotation off original star positions can be totally eliminated.

The part above presented the effectiveness of the algorithm in idealized condition. It can be inferred that if Equation (4) is used to calculate the feature of images with noise, the spots with grayscale more than 0 will increase a lot. And this won't accord with the real situation. To solve the problem, the algorithm adopted a limiting principle that only if the pixel has the grayscale value more than that of Grade 7 star, its grayscale will be included to calculate the feature of star image. That means adding a constraint condition $G(j) \geq 255 / 2.51^{7-1}=1.01976$ to Equation (4). In observed star images with noise, the result of pattern identification may be that several guide templates all satisfy the constraint condition set by the algorithm. At that time, the algorithm will choose the guide template which has the least difference in feature and central pixel's magnitude with observation template.

\section{Experimental results}

We generated 1000 directions of visual axis randomly to test the success rate of main star identification. In the condition where grayscale value noise of 0.1 units standard deviation and location noise of 0.5 pixels standard deviation (noise referred to in this paper are all Gaussian noise) were added to observed star images, the rate that the only main star of each observed star image was recognized successfully is $95.7 \%$.

\section{Performance on eliminating error}

Seen from the simulation result, the algorithm's performance to eliminate error caused by rotation in captured star image is obvious. For example, Figure 5 is an image simulated as observed star image containing grayscale value noise of 0.1 units standard deviation and location noise of 0.5 pixels standard deviation. By the algorithm in this paper, we got the result of star image identification, which was that the only main star in Figure 5 is the 1800th star in guide star catalog. When we set the direction of visual axis as the direction of that $\operatorname{star}^{[6]}$, we got the guide template corresponding to the observed image, as is illustrated in Figure 6. 


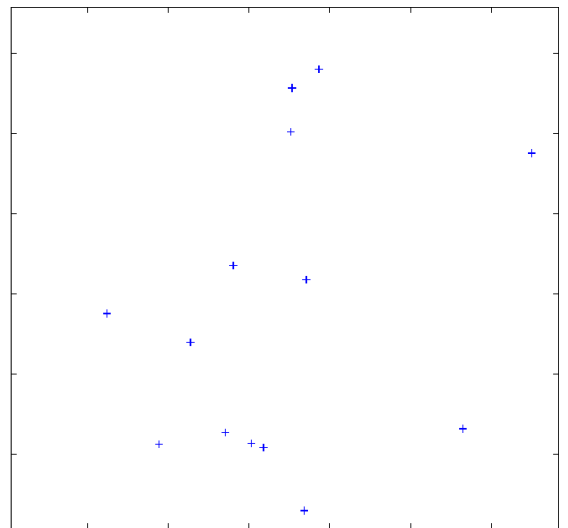

Figure 5 observed image

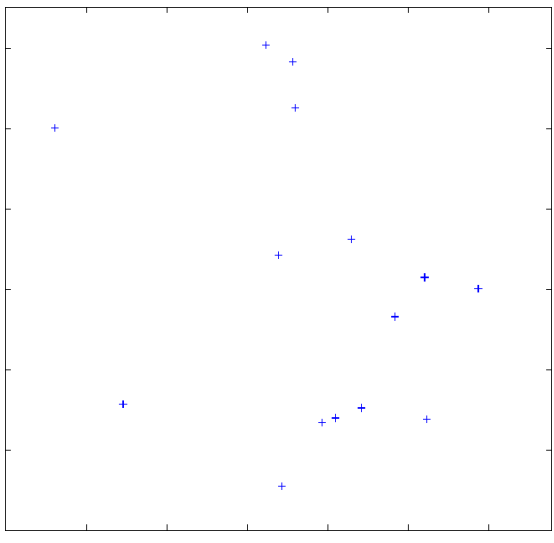

Figure 6 result of identification

We can see that the two images are almost opposite to each other, however, the result of match is accurate. Actually there was no procedure of rotation in the course of star pattern recognition, but the result showed that the algorithm could identify the main star correctly even there was a large extent of rotation between the observed image and guide template.

\section{performance comparison}

The performances of triangle algorithm, match group algorithm, grid algorithm and the algorithm in this article are shown in Table $1^{[7][8]}$. The operation condition of the three algorithms is the same with that of the algorithm presented in this paper.

Table 1 Performance comparison of various identification algorithms

\begin{tabular}{ccc}
\hline Algorithm & Run time(s) & Memory requirement $(\mathrm{Mb})$ \\
\hline Triangle & 1.8 & 3 \\
Match group & 1.6 & 0.7 \\
Grid & 0.04 & 0.5 \\
Method of this article & 0.06 & 0.076 \\
\hline
\end{tabular}

We can see that the memory cost of this algorithm is a lot less compared with the other three algorithms in Table 1. That's because it only needs space to store the features of different guide templates and magnitudes of guide stars.

In Figure 7, four curves represent the percentage of star fields identified over 500 random orientations of the sensor. The location error was held constant with a standard deviation of 0.5 pixels. The standard deviation for the brightness error is shown along the horizontal axis in units' stellar magnitude.

In Figure 8, four curves represent the percentage of star fields identified over 500 random orientations of the sensor. The brightness error was held constant with a standard deviation of 0.3 units' stellar magnitude. The standard deviation for the location error is shown along the horizontal axis in pixels.

\section{Conclusions}

This article presented algorithm for star image identification uninfluenced by rotation. The feature defined in the algorithm eliminates the influence from rotation error. Thus the procedure for image processing before pattern recognition can be eliminated. That's an advantage compared with traditional algorithms of star image identification. The result of simulation indicated the 
performance of this algorithm against location error and magnitude error is excellent and the time and storage cost for identification is optimized.

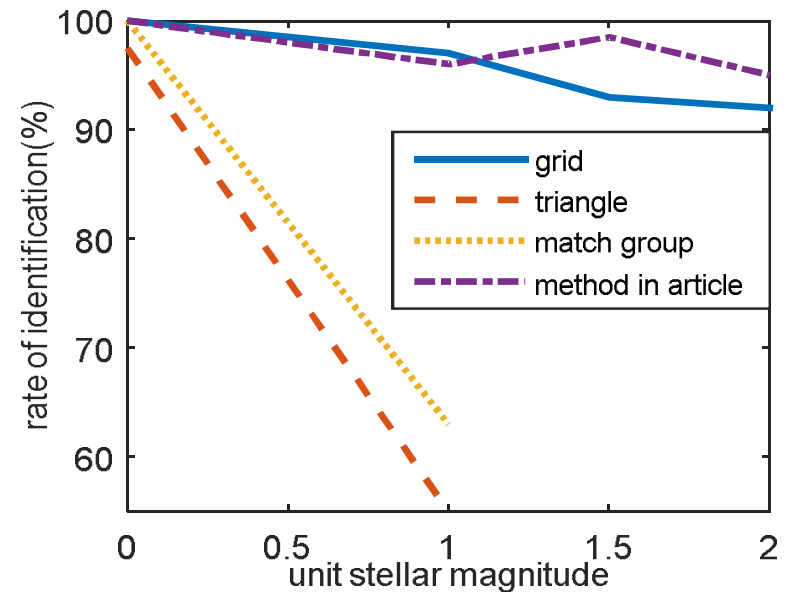

Figure 7 Rate of identification with several methods with magnitude error changing

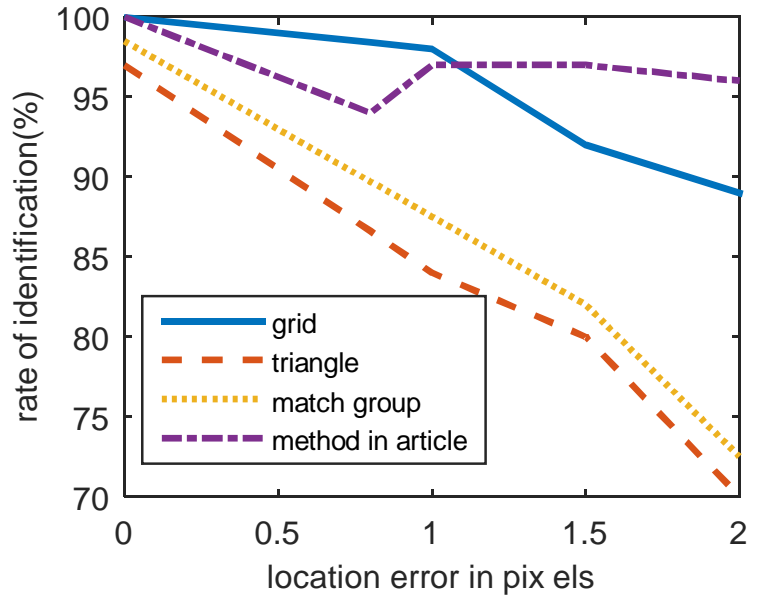

Figure 8 Rate of identification with several methods with location error changing

\section{References}

[1] Zhang Lei, He Xin, Wei Zhonghui, Liu Yanjun, and Guo Jingming. Modification of triangle identification algorithm [J]. Optics and precision engineering. 2010, (2): 458-463.

[2] Lin Tao, Zhou Jianli, Zhang Junping, Jia Xiaoguang, and Qian Guohui. All-sky automated quaternary star pattern recognition [J]. Journal of astronautics. 2000, 21(2) :82-85.

[3] Zhang Shaodi, Wang Yanjie, and Sun Hongha. Application of triangulation and RBF neural network to star pattern recognition[J]. Optics and precision engineering. 2012, (2): 395-402. [4] LI Li-hong , XU Hong-ze, ZHANG Fu-en. A kind of improved grid algorithm for all-sky autonomous star field identification [J], Optical Technique, 2000, 26(3): 204-210.

[5] Wei Xin-guo, Zhang Guang-jun, Jiang Jie. A star map identification algorithm using radial and cyclic features [J], Opto-Electronic Engineering, 2004, 31(8): 4-7.

[6] Padgett C, Kreutz-Delgado K, Udomkesmalee S. Evaluation of star identification techniques[J], Journal of Guidance, Control and Dynamics, 1997, 20(2): 259-267.

[7] M. S. Scholl. Star-Field Identification for Autonomous Attitude Determination [J]. Journal of Guidance, control and dynamics, 1995,18(1): 61-65.

[8] K.Ho. A survey of algorithms for star identification with low-cost star trackers [J]. Acta astronautica. 2012, (73): 156-163. 